\title{
First-principles investigation of carbon nanotube capacitance
}

\author{
Pawel Pomorski, ${ }^{1,2}$ Christopher Roland, ${ }^{2}$ Hong Guo, ${ }^{1}$ and Jian Wang ${ }^{3}$ \\ ${ }^{1}$ Center for the Physics of Materials and Department of Physics, McGill University, Montreal, PQ, Canada H3A 2T8 \\ ${ }^{2}$ Department of Physics, The North Carolina State University, Raleigh, North Carolina 27695-8202 \\ ${ }^{3}$ Department of Physics, The University of Hong Kong, Pokfulam Road, Hong Kong, China
}

(Received 10 February 2003; published 30 April 2003)

\begin{abstract}
With $a b$ initio simulations based on a real-space nonequilibrium Green's function formalism, we have investigated the charging of carbon nanotube systems. The charging effects are described by capacitance coefficients, for which we provide a first-principles estimate. Specifically, the capacitance matrix of nested armchair nanotubes, the insertion of one nanotube into another, and a junction of two metallic nanotubes with a large conductance gap were calculated with a focus on investigating the bias-induced charges. For the case of the nanotube junction, the numerical value of the capacitance is sufficiently high, as to be useful for future device applications.
\end{abstract}

DOI: 10.1103/PhysRevB.67.161404

PACS number(s): 72.80.Rj, 73.61.Wp

Depending on their helicity, carbon nanotubes are either metals or semiconductors, which makes them an ideal material for exploring quantum transport at the nanometer length scale. ${ }^{1}$ With the aid of nanomanipulators, prototypical nanotube-based devices have already been created in the laboratory, and their properties explored both experimentally ${ }^{2}$ and theoretically. ${ }^{3}$ Most of these investigations have focused on the conductance of nanotube devices. To date there are, however, very few investigations of other important transport properties such as the capacitance and self-inductance, which are properties that depend on the induced rearrangements of charge, rather than on the direct flow of current. For general nanoscale systems, it has been theoretically predicted ${ }^{4}$ and experimentally verified ${ }^{5}$ that capacitance of molecular scale conductors shows distinct nonclassical behavior, as previously anticipated from general considerations. ${ }^{6}$ Currently, there is little or no understanding of capacitive issues for nanotube-based systems, even though these determine the ability of nanotubes to charge-up and store charge, as well as providing important physics concerning the dynamic response of nanotubes to external ac fields. ${ }^{7,8}$ A thorough understanding of nanotubes as scanning probes, memory cells, and nanoelectronic devices requires a good knowledge of charge distribution and capacitance under an external bias potential. ${ }^{9}$

To address these issues, we present the results of an $a b$ initio investigation of the quantum capacitance of prototypical carbon nanotube systems. Specifically, we investigate the capacitance behavior of two armchair nanotube shells, the insertion of one nanotube into another, and a metal/metal nanotube junction. At the nanoscale, when the screening length is comparable to the dimensions of the system, the classical concept of electrostatic capacitance is no longer adequate. ${ }^{6}$ Instead, one must use the notion of electrochemical capacitance, $C_{\alpha \beta}=e d Q_{\alpha} / d \mu_{\beta}$, which measures the charge variation $d Q_{\alpha}$ on conductor $\alpha$ when the electrochemical potential of the reservoir connected to conductor $\beta$ is changed by a small amount $d \mu_{\beta} \cdot C_{\alpha \beta}$ takes quantum effects into account, and may behave qualitatively and quantitatively very differently when compared to its classical counterpart. $^{4-6}$
To calculate the capacitance of nanotube-nanotube junctions, we made use of an $a b$ initio analysis based on the Keldysh nonequilibrium Green's (NEGF) functions combined with density-functional theory (DFT). Our NEGF-DFT computation package, McDCAL, has been extensively described elsewhere, ${ }^{10,11}$ and has the advantage of being able to calculate the charge density for open quantum systems under a bias voltage entirely self-consistently, at least at a DFT level. The important contributions made by the bound states $^{13}$ present in the system are also included naturally. Here, we focus on calculating the charge when a finite bias is applied to the reservoirs [i.e., $\Delta Q=Q(V+\Delta V)-Q(V)$ ], and then directly calculate capacitance from its definition with $\Delta V=e d \mu$. As a further feature, we have used the Dirichlet boundary conditions for the electrostatic potential at the walls of our finite-sized calculational box, which corresponds to the whole system being surrounded by a metal container. ${ }^{14}$ This serves to terminate any field lines that escape from the nanotubes. When the container becomes infinite in size, the results reduce to that of a nanotube system in free space. The advantage of this approach is that it allows for the treatment of charged nanotube systems. The main quantities calculated are

$$
\begin{aligned}
& \Delta Q_{1}=C_{11} \Delta V_{1}+C_{12} \Delta V_{2}, \\
& \Delta Q_{2}=C_{21} \Delta V_{1}+C_{22} \Delta V_{2},
\end{aligned}
$$

with coefficients $C_{\alpha \alpha}$ giving the "self-charging" and $C_{\alpha \beta}$ the "mutual-charging" between the conductors.

Nested armchair nanotubes. As a first example, we calculate the capacitance per unit length of two nested, armchair nanotubes. This system, which corresponds to that of two multiwalled nanotube shells, has recently been realized experimentally. ${ }^{15}$ Specifically, we examined the case of a metallic $(5,5)$ nanotube (conductor 1$)$ inside a larger $(m, m)$ nanotube (conductor 2 ), with helicity index $m$ running from 12 to 22 . For the smallest $(12,12)$ outer tube, the closest distance between atoms in the inner and outer tubes is $9.1 \AA$, so that there is only a negligible overlap between the orbitals of the two tubes. Thus, although the real-space potential is 


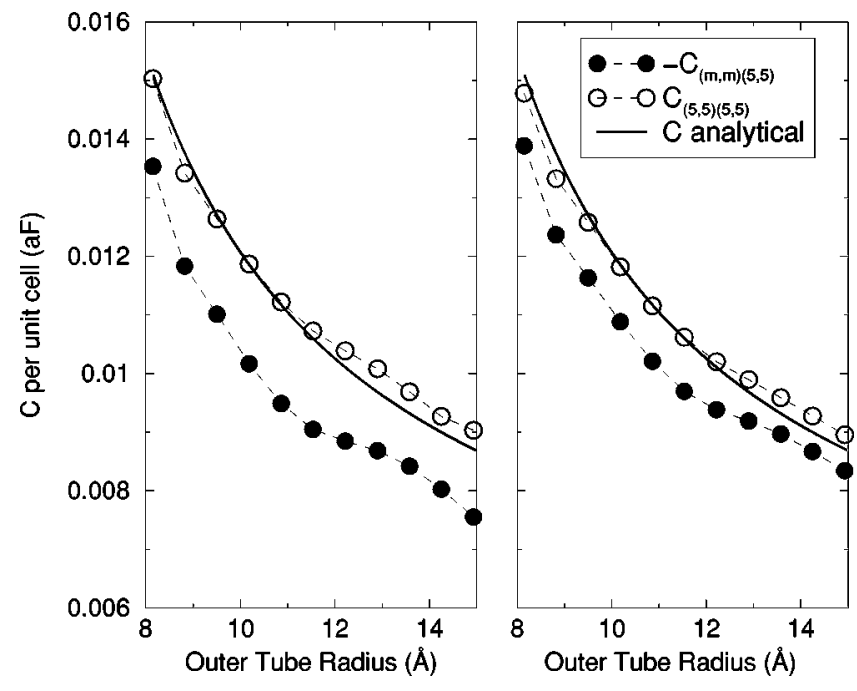

FIG. 1. Capacitance vs tube radius for $(5,5)$ shell inside $(\mathrm{m}, \mathrm{m})$ tubes. The left (right) panel shows data for a $40 \times 40 \AA(80$ $\times 80 \AA)$ metal container enclosing the system, respectively. The analytical result obtained via Eq. (3) is shown as a solid line.

coupled, the two tubes are de facto separated, with no dc current flowing between them. By connecting these tubes to two different reservoirs, a quantum system is constructed that is analogous to the case of a classical, concentriccylindrical capacitor. The calculated capacitive response is linear (to better than 1\%), at least up to $5 \mathrm{~V}$ so that a welldefined voltage-independent capacitance is obtained. This is reasonable, and reflects the relatively large window of constant DOS around the Fermi level for armchair nanotubes.

Consider the case of a $(5,5) /(12,12)$ nanotube system, all inside a metal container of $40 \times 40 \AA$ in the plane perpendicular to the nanotube axis. We obtain the capacitance coefficients per unit cell as $C_{11} \equiv C_{(5,5)(5,5)}=0.0150$ $\mathrm{aF}, \quad C_{21} \equiv C_{(12,12)(5,5)}=-0.0135 \mathrm{aF}, \quad C_{12} \equiv C_{(5,5)(12,12)}$ $=-0.0134 \mathrm{aF}$, and $C_{22} \equiv C_{(12,12)(12,12)}=0.0243 \mathrm{aF}$. If the metal box were to be made infinitely large, the magnitude of all four coefficients would be the same. For a finite-sized metal container, however, some of the induced charge will be located on the container. Thus, the coefficient $C_{(12,12),(12,12)}$ can be expected to be larger because it will have an increased coupling to the boundary. In response to the potential variations on the $(12,12)$ tube, the induced charge on the $(5,5)$ tube amounts to only $55 \%$ of the charge on the $(12,12)$ tube, indicating that the rest of the induced charge is on the container. In contrast, if the charge is injected into the $(5,5)$ tube, then $89 \%$ of the induced charge is located on the $(12,12)$ tube, which acts as a reasonably efficient screen. Note also that $C_{(12,12)(5,5)}$ and $C_{(5,5)(12,12)}$ agree with each other to better than $1 \%$. In the infinite container limit, all the coefficients take on a value of $\sim 0.015 \mathrm{aF}$. Figure 1 shows $C_{(5,5)(5,5)}$ and $C_{(m, m)(5,5)}$ for different armchair tubes as a function of their radius, for two different container sizes: $40 \times 40 \AA$ and 80 $\times 80 \AA$. As the metal container gets larger, the outer tube screens more of the charge on the inner tube, so that the numerical value of the two coefficients becomes closer. As expected, the value of $C_{(5,5)(5,5)}$ changes only slightly as the box size increases.
It is particularly interesting to probe the classical limit for this nanotube system. Following the Büttiker formalism, ${ }^{6}$ it is possible to derive a simple analytical formula describing the capacitive behavior. The system geometry consists of two thin, concentric cylindrical tubes of length $l$, modeled as sheets of a two-dimensional electron gas. It is assumed that these tubes do not interact with the boundary container, and that the system is always neutral. All capacitive coefficients will therefore have equal magnitude $C$, whose value is given by $^{12}$

$$
\frac{2 \pi \epsilon_{o} l}{C}=\ln \left(\frac{R_{2}}{R_{1}}\right)+\frac{\lambda_{1}}{R_{1}}+\frac{\lambda_{2}}{R_{2}} .
$$

Here $R_{1,2}$ are the radii of the outer and inner tubes, $\lambda^{-1}$ $=4 \pi e^{2} d \sigma / d E$ is the screening length, and parameter $d \sigma / d E$ is the DOS per unit area of the respective cylindrical tubes. To compare Eq. (3) with our nanotube results, we estimate the electronic parameters $\lambda, d \sigma / d E$ using our $a b$ initio data; the quantum correction terms $\lambda_{1,2} / R_{1,2}$ are found to have a constant value of $\sim 0.15$ for the armchair nanotubes considered here. The geometric parameters $R_{1,2}$ are estimated as the average radius of the charge distribution of each nanotube, and take on values of $(8.14-0.54) \AA$ for the outer $(12,12)$ and $(3.39+0.73) \AA$ for the inner tube, respectively. Using parameters determined in this way, one can estimate all the capacitive coefficients. As shown in Fig. 1, the agreement between our $a b$ initio data and the simple formula is quite good. Note that for this nested nanotube system, the quantum correction terms $\lambda / R$ are comparable to the classical logarithmic term. Clearly, a naive calculation of the coefficients based on classical considerations would not be adequate.

Inserted nanotubes. Next, we examine the capacitance of a two-probe system in which a capped $(5,5)$ nanotube is inserted a finite distance into an open $(12,12)$ tube, with the central axis of the two tubes coinciding, as shown in Fig. 2. The system now consists of two semi-infinite carbon nanotube leads and a central region containing the junction of the two tubes. As in the previous case, the entire system is surrounded by a metal container at a specified gate voltage. Details of the charging of the nanotubes is shown directly in Fig. 2, along with a histogram showing the change in the total charge accumulated on each nanotube ring. In particular, note that the $(12,12)$ tube acquires a very large amount of charge on its terminal ring, which is most likely due to the presence of its dangling bonds.

For this situation, we also found that the charge accumulation is essentially linear for a bias of at least up to $1 \mathrm{~V}^{16}$ The calculated values of the capacitive matrix are $C_{(5,5)(5,5)}$ $=0.1050 \mathrm{aF}, \quad C_{(12,12)(5,5)}=-0.0455 \mathrm{aF}, \quad C_{(5,5)(12,12)}$ $=-0.0451 \mathrm{aF}$, and $C_{(12,12)(12,12)}=0.1565 \mathrm{aF}$, which all have similar characteristics to the previously discussed case of nested nanotube shells. Figure 2(e) shows $C_{(12,12)(5,5)}$ as a function of the nanotube insertion depth. In the limit of large insertion, the increase in capacitance per unit length should match the capacitance per unit length obtained for the double-wall nanotube configuration discussed above. Indeed, we measure a capacitance of $0.012 \mathrm{aF}$ from the slope of 


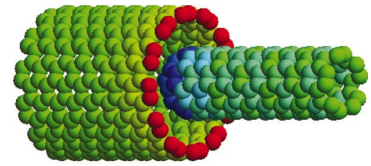

(a)

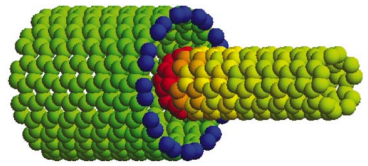

(b)
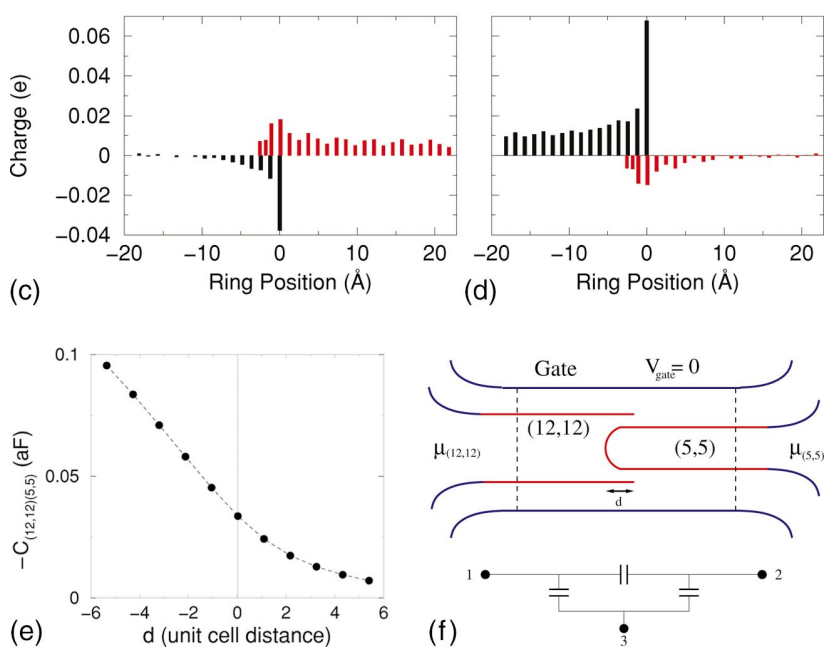

FIG. 2. (Color) (a-d) Tube charging of the $(12,12) /(5,5)$ biased junction for a central simulation box with 458 atoms. A $+0.272 \mathrm{~V}$ bias is applied on the right $(5,5)$ tube in $(\mathrm{a}, \mathrm{c})$, and on the left $(12,12)$ tube in $(\mathrm{b}, \mathrm{d})$. Upper panels $(\mathrm{a}, \mathrm{b})$ show charging with accumulation shown with a color scheme (blue corresponding to charge addition and red to charge depletion). Lower panels (c,d) display a histogram plot of the charge accumulated on the tube rings, with the black (red) bars showing charge accumulation on the $(12,12)$ [capped $(5,5)]$ tubes, respectively. Panel (e) shows $C_{(12,12)(5,5)}$ as a function of the relative position $d$ of the two nanotubes. The origin $d=0$ is chosen such that the longitudinal positions of the terminal rings of both tubes coincide, and the negative $d$ indicates overlap between tubes. Panel (f) illustrates the computational configuration and equivalent device circuit.

Fig. 2(e), which is in reasonable agreement with two-shell result, given both the finite size of our box and the capped structure of the $(5,5)$ tube considered here. From Figs. 2(c,d), it is also evident that the nanotube to which the bias is applied gains charge along its entire length in the junction region. This "self-charging" is due to the capacitive coupling between the nanotube and the surrounding metal container. It therefore follows that the values of $C_{(m, m)(m, m)}$ calculated via Eq. (2) will increase linearly in size, as more and more charge density is included. To understand this, it is useful to consider the equivalent circuit for this device, shown in Fig. 2(f). In addition to the capacitance between the nanotubes in the central cell, there are additional capacitance due to the lead nanotubes and the gate container interactions. These capacitances must be proportional to the length of the nanotube leads. For a system enclosed in an infinitely large box, the capacitive coupling between the lead/box will vanish, and all charge variations will occur in the vicinity of the junction only. Again, all capacitance matrix coefficients would then be equal in magnitude.

Metallic nanotube junction. As a final example, we consider the capacitance of a $(12,0) /(6,6)$ nanotube junction, in

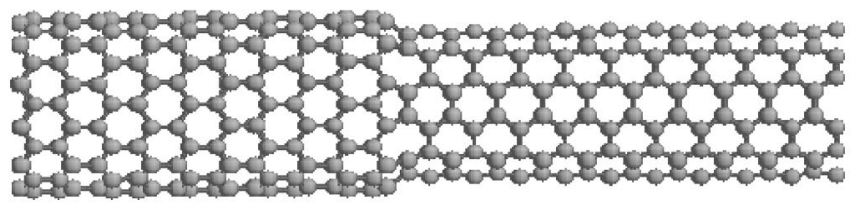

(a)

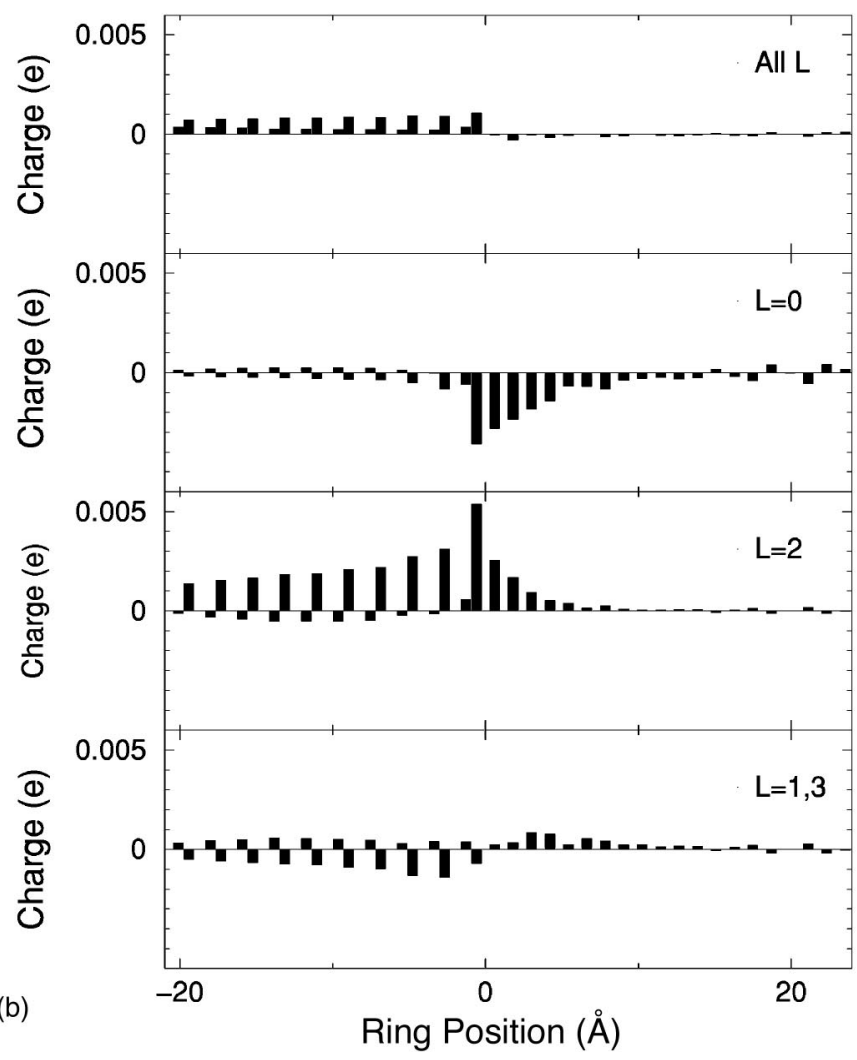

FIG. 3. Panel (a) shows the 480 atom central simulation box we used to obtain the capacitance coefficient values for the $(12,0) /(6,6)$ junction. Panel (b) shows the total and symmetry decomposed variation in charge on nanotube rings, under +0.272 bias applied to the left $(12,0)$ tube.

which the two nanotubes are joined seamlessly to each other, shown in Fig. 3(a). This junction is characterized by a large conductance gap about the Fermi energy: because of the symmetry of the tubes, electrons arriving at the junction undergo total reflection and no current flows through the junction. $^{3}$

In this contact junction, we separate the charge variation due to electrons arriving from each tube by dividing the electron density into contributions from electrons with wave function symmetries $\{L=0,1,2,3\}$ under rotation group $C_{6}$. Here $L$ denotes the number of nodes of the wave functions around tube circumference. Specifically, given the symmetry of the bands near the Fermi energy, the $L=0$ symmetry contribution gives the charge arriving from the $(6,6)$ tube, while the $L=2$ symmetry gives the contribution from $(12,0)$ tube. $^{3}$ Charge due to the $L=1,3$ symmetries describe electrons arriving via other sub-bands, and therefore represent a polarization response. These contributions, as well as the variation of total charge density, are shown in Fig. 3(b) for the case of bias applied on the $(12,0)$ tube. Because the tubes are in 
contact, the charge contributions overlap due to the overlap of the $L=0,2$ DOS. The net charge of the $L=1,3$ symmetries is two orders of magnitude smaller than the charge accumulated in the $L=0,2$ symmetries, consistent with a picture of polarization response. The capacitance coefficients can be obtained by integrating the $L=0$ or $L=2$ charge accumulations. The small-bias capacitance coefficients are found to be $^{17} \quad C_{(6,6)(6,6)}=0.205 \mathrm{aF}, \quad C_{(12,0)(6,6)}=-0.125 \mathrm{aF}$, $C_{(6,6)(12,0)}=-0.127 \mathrm{aF}$, and $C_{(12,0)(12,0)}=0.199 \mathrm{aF}$. Here, the $C_{(6,6)(12,0)}$ coefficient, for instance, describes the charge arriving into the system from the $(6,6)$ reservoir $(L=0)$ in response to an electrochemical potential change in the $(12,0)$ nanotube reservoir. The self-capacitance coefficients are again larger because they include the self-charging contributions due to the lead/container interactions, as already discussed. Note that the numerical values of the capacitance matrix for this system are relatively high, as compared to the previously discussed $(5,5)(12,12)$ junctions. For the latter, a significant insertion distance was necessary in order to achieve similar values. For the $(12,0) /(6.6)$ junction, the capacitance is high, even though the tube contact area is significantly smaller. Thus, the overlapping of the DOS of the two tubes clearly boosts the capacitance significantly. Systems with such a high capacitance, in combination with zero current leakage because of the conductance gap, might well prove to be of future technological interest for nanoscale DRAM devices.

In summary, we have computed the finite-bias capacitance of several prototypical nanotube systems using a fully selfconsistent $a b$ initio approach. These calculations not only provide direct numerical estimates of the capacitance matrix, but also provide direct information as to the distribution of charge on nanotube systems. Furthermore, these calculations all point to the important role played by quantum corrections in determining the value of a nanoscale capacitance. The impact of these induced charge rearrangements on carbon nanotube-based devices will be left for future investigations.

We gratefully acknowledge Dr. Jeremy Taylor and Dr. Brian Larade for participating in the early stages of this work. We gratefully acknowledge financial support from NSERC of Canada (P.P. and H.G.), ONR and NASA (C.R.), and a RGC grant from the SAR Government of Hong Kong. We would like to thank the North Carolina Supercomputing Center (NCSC) for extensive IBM SP3 computer time.
${ }^{1}$ See, for example, M.S. Dresselhaus, G. Dresselhaus, and P.C. Eklund, Science of Fullerenes and Carbon Nanotubes (Academic Press, New York, 1996); J. Bernholc, C. Roland, and B. Yakobson, Crit. Rev. Solid State Mater. Sci. 2, 706 (1997).

${ }^{2}$ See, for example, S. Frank et al., Science (Washington, DC, U.S.) 280, 1744 (1998); A. Bezryadin et al., Phys. Rev. Lett. 80, 4036 (1998); J. Tans et al., Nature (London) 386, 474 (1997); M. Bockrath et al., ibid. 397, 598 (1998).

${ }^{3}$ See, for example, L. Chico, L.X. Benedict, S.G. Louie, and M.L. Cohen, Phys. Rev. B 54, 2600 (1996), and many other papers.

${ }^{4}$ J. Wang et al., Phys. Rev. Lett. 80, 4277 (1998).

${ }^{5}$ J.G. Hou et al., Phys. Rev. Lett. 86, 5321 (2001).

${ }^{6}$ M. Büttiker, J. Phys.: Condens. Matter 5, 9361 (1993); T. Christen and M. Büttiker, Phys. Rev. Lett. 77, 143 (1996).

${ }^{7}$ C. Roland, M. Buongiorno Nardelli, J. Wang, and H. Guo, Phys. Rev. Lett. 84, 2921 (2000).

${ }^{8}$ Y.P. Zhao et al., Phys. Rev. B 64, 201402 (2001).

${ }^{9}$ S.V. Kalinin et al., Appl. Phys. Lett. 81, 754 (2002); S.V. Kalinin et al., ibid. 81, 5219 (2002).

${ }^{10}$ J. Taylor, H. Guo, and J. Wang, Phys. Rev. B 63, 245407 (2001); J. Taylor, Ph.D. thesis, McGill University, 2000.

${ }^{11}$ In this work, we use $s, p$ LCAO real-space basis set, the exchange correlation is included at the LDA level, and we define the atomic core by standard pseudopotentials [D.R. Hamann, M. Schlüter, and C. Chiang, Phys. Rev. Lett. 43, 1494 (1982)].

${ }^{12}$ P. Pomorski, Ph.D. thesis, McGill University, 2002.

${ }^{13}$ Ryo Tamura and Masaru Tsukada, Phys. Rev. B 52, 6015 (1995).

${ }^{14}$ Ryo Tamura, Phys. Rev. B 64, 201404 (2001).

${ }^{15}$ J. Cumings and A. Zettl, Science (Washington, DC, U.S.) 289, 602 (2000).

${ }^{16}$ We also observe (Figs. 2 and 3) long-ranged Friedel-type oscillations that do not completely decay to zero within our central simulation box. Such oscillations are seen only in $d Q$; the equilibrium charge $Q$ does not display such oscillations. The presence of these oscillations and its imperfect decay away from tube interface does not affect the value of capacitance in any significant way. Specifically, the capacitance coefficients do not fluctuate due to the oscillations, as the size of the box is varied.

${ }^{17}$ The neutral $(12,0)$ nanotube is actually semimetal, with a small band gap of $0.082 \mathrm{eV}$. Since we are primarily interested in the metallic behavior of the tubes, we start by applying a constant voltage of $+3.0 \mathrm{~V}$ to both reservoirs connected to the two nanotubes. This shift results in a slight accumulation of charge ( $0.04 e$ per carbon atom) on both tubes, but was sufficient to shift the reference chemical potential $\mu$ away from the $(12,0)$ gap. 